\title{
Mathematical modeling for heat conduction in stone fruits
}

\author{
F. J. Cuesta ${ }^{1}$, M. D. Alvarez ${ }^{2 *}$
}

${ }^{1}$ Department of Products, Institute of Food Science, Technology and Nutrition (ICTANCSIC), José Antonio Novais, 10, 28040, Madrid, Spain

${ }^{2}$ Department of Characterization, Quality, and Safety, Institute of Food Science, Technology and Nutrition (ICTAN-CSIC), José Antonio Novais 10, 28040, Madrid, Spain

* Corresponding author.

E-mail address: mayoyes@ictan.csic.es (M. D. Alvarez)

Tel: +34 915492300

Fax: +34 915493627 


\begin{abstract}
Stone fruits are cooled after harvesting to extend their shelf life and prevent postharvest losses. Because they are quickly subject to chilling injuries at inappropriate temperatures, their thermal properties should be known in order to design an optimum cooling process. However, how long does it take for an olive to reach its storage temperature at the stone-pulp interface? This paper proposes approximated equations as a model for predicting cooling times at the stone-pulp interface and for measuring the thermal diffusivity of the pulp and the external heat transfer coefficient. The model is based on a solution in Fourier series for the conduction of heat in spheres with an inner concentric, insulating spherical core, as a model of conduction of heat in stone fruits.
\end{abstract}

Keywords: Stone fruits; Heat transfer; Fourier series; Thermophysical parameters; Surface heat transfer coefficient. 


\section{Highlights}

A Fourier series solution for heat conduction in spherical stone fruits is proposed.

New equations for boundary equation and constants of the series are deduced.

New asymptotic approximations to boundary equation first eigenvalue are proposed.

Cooling power needed to cool the fruit is deduced. 


\section{Introduction}

In postharvest processing of plant foods, rapid cooling is generally necessary to extend their shelf life and reduce losses (Erdoğdu et al. 2014). In the design of this processing there are basic control parameters that it is necessary to predict accurately, such as the cooling time needed to reach a certain temperature at the thermal center of the product, the average temperature of the product at a given time, the amount of heat to be extracted to reach that temperature, etc. In most cases, the calculation of these parameters is based on the first approximation to the general Fourier series solution for regular geometries in homogeneous isotropic bodies, which usually does not explicitly include the stone. In addition, the indirect measurements of thermal diffusivity and the external heat transfer coefficient are also based on these approximations (Awuah et al. 1995; Erdoğdu 2005, 2008). It is obvious, therefore, that it would be useful to have appropriate mathematical models that explicitly include the stone, to allow accurate prediction of the design parameters mentioned above, and to measure the thermal diffusivity and the film coefficient.

In fact, among the analytical solutions to these problems in solids with elementary geometries there is a considerable body of literature on approximation models. Since the first published results in the 1960s (Gac 1963; Pflug et al. 1965; Smith et al. 1967), these models have been based on a linear approximation of the cooling kinetics (on a semi-logarithmic scale), valid from a given time onwards. The main applications of analytical approximations of this kind are: estimation of cooling/heating times, indirect measurement of the surface heat transfer coefficient $h$ when the thermophysical parameters are known, and measurement of thermal parameters if $h$ is known. In general, these approximation equations are valid for solids with elementary shapes 
(Becker and Fricke 2002; Erdoğdu 2005, 2008; Kondjoyan 2006). These approximate equations were extended to ellipsoidal or even irregular geometries in some works (Cleland and Earle 1982; Cuesta et al. 1990; Cuesta and Lamúa 1995, 2002; Lin et al. 1996a,b, 2000; Smith et al. 1967; Yilmaz 1995 (and the Letter to the Editor - about this last paper - published by Van Beek and Meffert (1997)).

In stone fruits, the theoretical thermal problems are different from those of solid products. In fact, stone fruits, such as olives, cherries, plums, and so forth, have approximately spherical or ellipsoid geometries, but inside they contain a ligneous core - the seed - whose physical and thermophysical parameters are radically different from those of the edible part, the pulp. Moreover, the contact surface between this seed and the pulp is, in practice, the deepest point that can be reached in the fruit and it performs the role of a "thermal center" which in homogeneous solid objects is represented by the geometric center. According to Cinquanta et al. (2002) and Di Matteo et al. (2000, 2002, 2003), the theoretical solution is easy to deduce, following the methodology described by Carslaw and Jaeger (1959), to which should be added the works by RuizLópez et al. (2004, 2007), which proposed analytical solutions for food drying kinetics, or the work by Helal (2012), who proposed an integral transform method for nonlinear heat-conduction problems in multilayered spherical media. Apart from these analytical works, we must resort to numerical calculation, where da Silva et al. (2009, 2010), Hernández-Díaz et al. (2008), Fabbri et al. (2011), Nilnont et al. (2012), Ruiz-López et al. (2011, 2012), Sabarez (2012), and Uyar and Erdoğdu (2012) have proposed algorithms developed to simulate the heat and mass transfer by numerical calculation in semi-spherical-geometry products and plums, respectively.

In spite of this, the literature contains little information that includes analytical solutions for heat transfer explicitly including the seed, and, consequently, the 
corresponding approximation equations for estimating the cooling time at the stonepulp interface, the energy required to reach that temperature, etc., or for determining the thermal diffusivity and the surface heat transfer coefficient (Biot number).

This paper is intended to be the first of a series of three consecutive ones. A second study takes into account the influence of the heat of respiration of the pulp, but also considers the adiabatic boundary condition at the stone-pulp interface. A third study will eliminate this condition, considering the stone as a concentric sphere with its own thermophysical constants but without internal generation of heat.

Accordingly, the first objective of this study was to present a solution in Fourier series for the kinetic temperature of spheres, explicitly considering a thermally insulating internal core, as a theoretical simulation of stone fruits. A second objective was to derive valid equations for the inverse simultaneous determination of thermal diffusivity and Biot number (dimensionless heat transfer coefficient), or to pre-calculate the aforementioned design parameters. As a practical example, the solution is applied to hydro-precooling of olives (Gordal variety).

\section{Mathematical Background}

\subsection{Governing equations and Fourier series solution}

\subsubsection{Temperature kinetics}

Consider a sphere of radius $R$ containing a concentric spherical kernel of radius $a$

(Fig. 1), which is to be considered quasi-insulating against the effects of heat transfer, as compared to external heat transfer, for the duration of the process. It was decided to 
adopt this condition in this paper as a consequence of having found practically no values for the thermophysical parameters of the drupe's stone, and of the fact that, in the cases we have examined, the stone has a woody layer with a porous appearance, in many cases externally similar to cork, protecting the seed inside. Therefore, it will be assumed that the initial temperature of the object, $T_{0}$, is uniform, and that it is placed in sudden contact with a mechanically stirred medium at a uniform temperature $T_{e x}$. Denoting the diffusivity of the object as $\alpha$, the time as $t$, and using the following dimensionless variables:

$$
Y=\frac{T-T_{e x}}{T_{0}-T_{e x}}, F O=\frac{\alpha t}{R^{2}}, x=\frac{r}{R}, x_{a}=\frac{a}{R}
$$

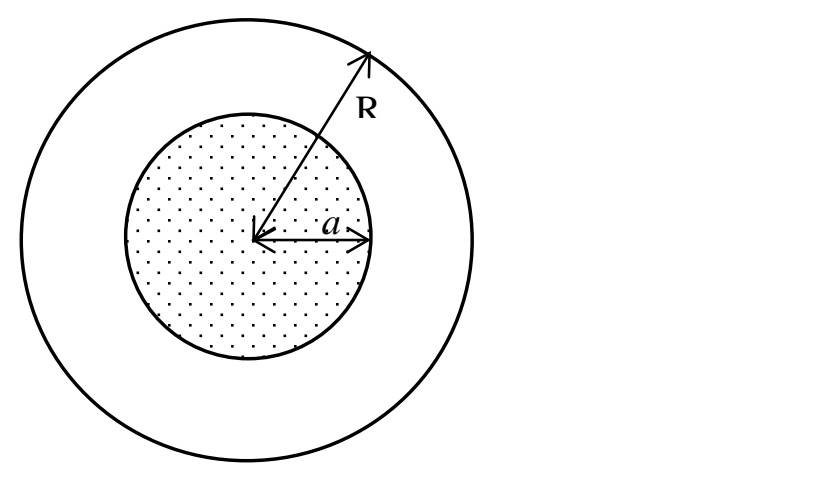

Fig. 1. Schematic of the model

The one-dimensional heat transfer equation with homogeneous isotropic constant coefficients, ignoring the internal heat source, can be written in dimensionless terms in the form (Carslaw and Jaeger 1959):

$$
\frac{1}{x^{2}} \frac{\partial}{\partial x}\left(x^{2} \frac{\partial Y}{\partial x}\right)=\frac{\partial Y}{\partial F O}
$$

with the initial condition:

$$
F O=0: Y_{0}=1
$$


and the following boundary conditions:

A) External surface: Boundary conditions of the third kind:

$$
\text { Fo }>0 ;\left[\frac{\partial Y}{\partial x}\right]_{x=1}=-B i Y_{S f}
$$

$B i$ being the Biot number.

B) The sphere/kernel contact surface:

$$
F O>0 ; x=x_{a}:\left[\frac{\partial Y}{\partial x}\right]_{x=x_{a}}=0
$$

The general solution can be expressed as in the case of a homogeneous solid sphere as a Fourier series in separate variables (see Appendix A):

$$
Y(x, F O)=Y=\sum A_{n} \psi\left(\delta_{n} x\right) e^{-\delta_{n}^{2} F o}
$$

And its mass average value

$$
\begin{aligned}
& \bar{Y}(F o)=\bar{Y}=\sum \bar{A}_{n} e^{-\delta_{n}^{2} F o} \\
& \psi\left(\delta_{n} x\right) \text { being: } \\
& \psi\left(\delta_{n} x\right)=\frac{\sin \left[\delta_{n}\left(x-x_{a}\right)\right]+\delta_{n} x_{a} \cos \left[\delta_{n}\left(x-x_{a}\right)\right]}{\delta_{n} x}
\end{aligned}
$$

$\delta_{n}$ are the roots of the transcendental boundary equation and $A_{n}$ are the constants of the series expansion, whose values in the general case are, respectively (see Appendix B):

$$
\begin{aligned}
& \delta_{n}=\tan \left[\delta_{n}\left(1-x_{a}\right)\right] \frac{1-B i+\delta_{n}^{2} x_{a}}{1+x_{a}(B i-1)} \\
& A_{n}=\frac{2 B i \psi\left(\delta_{n}\right)}{\left[\psi\left(\delta_{n}\right)\right]^{2}\left(\delta_{n}{ }^{2}+B i^{2}-B i\right)-\delta_{n}{ }^{2} x_{a}^{3}}
\end{aligned}
$$

When $B i \rightarrow \infty$ Eqs. (9) and (10) become:

$$
\begin{aligned}
& \tan \left[\delta_{n, \text { Max }}\left(1-x_{a}\right)\right]+\delta_{n, \text { Max }} x_{a}=0 \\
& A_{n, \infty}=\frac{-2}{\cos \left[\delta_{n, \operatorname{Max}}\left(1-x_{a}\right)\right]} \frac{1}{\left(1+\delta_{n, \text { Max }}^{2} x_{a}^{2}-\delta_{n, \text { Max }}^{2} x_{a}^{3}\right)}
\end{aligned}
$$


And

$$
\psi\left(\delta_{n, \operatorname{MAx}}\right)=\frac{\sin \left[\delta_{n, \operatorname{Max}}\left(1-x_{a}\right)\right]+\delta_{n, \operatorname{Max}} \cdot x_{a} \cos \left[\delta_{n, \operatorname{Max}}\left(1-x_{a}\right)\right]}{\delta_{n, \operatorname{Max}}}=0
$$

Where the case $B i \rightarrow \infty$ is identified by the subscript $\infty$, except for $\delta_{n, \text { Max }}$, which is identified by the subscript "Max" to indicate that it is the maximum value that can be achieved (for $B i \rightarrow \infty$ ). As can be seen, these equations explicitly include the relative coordinate of the nucleus. Furthermore, equations (8) through (13) reduce to those of the solid sphere when $x_{a}=0$.

For the mass average the constants are:

$$
\begin{aligned}
& \bar{A}_{n}=A_{n} \bar{\psi}\left(\delta_{n}\right)=\frac{6 B i^{2}}{\delta_{n}^{2}\left(1-x_{a}^{3}\right)} \cdot \frac{\left[\psi\left(\delta_{n}\right)\right]^{2}}{\left[\psi\left(\delta_{n}\right)\right]^{2}\left[\delta_{n}^{2}+B i^{2}-B i\right]-\delta_{n}^{2} x_{a}^{3}} \\
& \bar{A}_{n, \infty}=A_{n, \infty} \bar{\psi}\left(\delta_{n, \text { Max }}\right)=\frac{6}{\delta_{n, \text { Max }}^{2}\left(1-x_{a}^{3}\right)} \frac{1+\delta_{n, \text { Max }}^{2} x_{a}^{2}}{\left(1+\delta_{n, \text { Max }}^{2} x_{a}^{2}-\delta_{n, \text { Max }}^{2} x_{a}^{3}\right)}
\end{aligned}
$$

Fig. 2 shows the first eigenvalue $\left(\delta_{1}\right)$ of boundary Eq. (9) vs. $B i$ for $x_{a}=0.3$ to 0.6 , and solid sphere as reference. The reason for this range is that, according to Cinquanta et al. (2002), Hernández et al. (2010), and Jiménez-Jiménez et al. (2013), it is the average diameter ratio range of the stone. These values were confirmed in an extensive work on prevention of browning of two varieties of table olives by pre-cooling in cold water, carried out at the Institute of Food Science, Technology and Nutrition (ICTANCSIC). The data used later in the practical examples in this paper are part of it. 


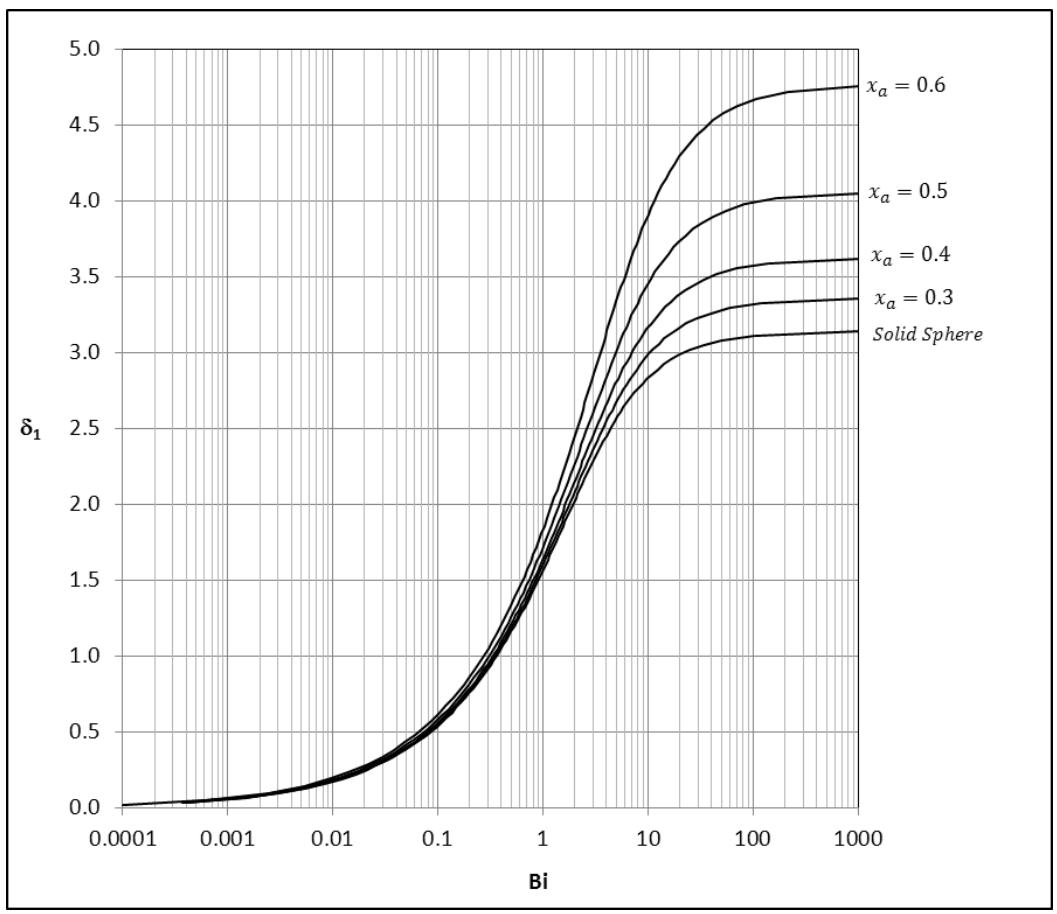

Fig. 2. First eigenvalue $\left(\delta_{1}\right)$ of boundary equation (9) vs. $B i$ for $x_{a}=0.3$ to 0.6 , and solid sphere as reference.

\subsubsection{Heat flow kinetics: total heat exchanged with the medium}

The heat flow transferred by the fruit through the entire surface at moment $t$ is (Newton's law):

$$
\dot{Q}=-4 \pi R^{2} h\left(T_{s f}-T_{e x}\right)=-4 \pi R^{2} h \Delta T_{0} \sum A_{n} \psi\left(\delta_{n}\right) e^{-\delta_{n}^{2} F o}
$$

Or, denoting by $\dot{Q}_{0}$ the total initial heat flow transferred by the fruit through its surface:

$$
\begin{aligned}
& \dot{Q}_{0}=4 \pi R^{2} h \Delta T_{0} \\
& \dot{Q}=-\dot{Q}_{0} \sum A_{n} \psi\left(\delta_{n}\right) e^{-\delta_{n}^{2} F o}
\end{aligned}
$$

So the total energy extracted from the fruit up to moment $t$ is (by integrating Eq. (17) and rearranging terms):

$$
Q(t)=\int_{0}^{t} \dot{Q} d t^{\prime}=\dot{Q}_{0} \sum \frac{A_{n}}{\delta_{n}^{2}} \psi\left(\delta_{n}\right)\left(e^{-\delta_{n}^{2} F o}-1\right)
$$


The average flow transferred by the fruit until time $t$ is:

$$
\overline{\dot{Q}}(t)=\frac{1}{t} \int_{0}^{t} \dot{Q} d t^{\prime}=\frac{\dot{Q}_{0}}{F o} \sum \frac{A_{n}}{\delta_{n}^{2}} \psi\left(\delta_{n}\right)\left(e^{-\delta_{n}^{2} F o}-1\right)
$$

The maximum amount of energy removable from the fruit $(t=\infty)$ at $B i=$ Const. and with the initial temperature difference $\Delta T_{0}$ is:

$$
Q_{M}=\frac{\dot{Q}_{0}}{a} R^{2} \sum \frac{A_{n}}{\delta_{n}^{2}} \psi\left(\delta_{n}\right)
$$

And the absolute maximum amount of heat extractable from the fruit for $t=\infty$ and $B i=\infty$ is:

$$
Q_{M, B i \rightarrow \infty}=\frac{K}{a} R^{2} \sum \frac{A_{n, \infty}}{\delta_{n, \text { Max }}^{2}} \frac{-1}{\sin \left[\delta_{n, \operatorname{Max}}\left(1-x_{a}\right)\right]}
$$

\subsection{First approximations and applications}

\subsubsection{Kinetics of temperature}

As in the case of the homogeneous solid sphere, from a given moment onwards the infinite series converges very quickly, because from the second exponential factor onwards its value also decreases very quickly, so in practice its contribution to the final sum of the complete series is null. Thus, for practical purposes, exact analytical solutions lead to first-approximation models based on a linear approximation of the cooling graph (on a semi-logarithmic scale), which are valid from a given time. Apart from estimating the cooling/heating times, the main applications of these linear approximations are in indirect measurement of thermophysical parameters (thermal diffusivity and conductivity), and the surface heat transfer coefficient. In dimensionless terms, the first term of the complete series at the thermal center (stone-pulp contact) is:

$$
Y(F o)=Y \approx A_{1} e^{-\delta_{1}^{2} F o}
$$

Or: 
$\ln Y \approx \ln A_{1}-\delta_{1}^{2} F o=\ln A_{1}-S \cdot t$

$S \equiv \frac{a \cdot \delta_{1}{ }^{2}}{R^{2}}$

$A_{1}$ being the experimental lag factor and $\delta_{1}$ the first eigenvalue of the boundary condition (Eq. (9)).

\subsubsection{Kinetics of thermal flow}

In the same manner as in Eq. (21) for the kinetic temperature, the estimation of the heat flow and the amount of energy removed from the fruit up to moment $t$ can be approximated by the first term of Eqs. (17) and (18):

$$
\dot{Q} \approx-\dot{Q}_{0} A_{1} \psi\left(\delta_{1}\right) e^{-\delta_{1}^{2} F o}
$$

and

$$
\begin{aligned}
& Q(t) \approx \dot{Q}_{0} \frac{A_{1}}{\delta_{1}^{2}} \psi\left(\delta_{1}\right)\left(e^{-\delta_{1}{ }^{2} F o}-1\right) \\
& \dot{Q}_{0}=4 \pi R^{2} h \Delta T_{0}
\end{aligned}
$$

And the time average power needed to cool the fruit:

$$
\overline{\dot{Q}}(t) \approx \frac{\dot{Q_{0}}}{F_{o}} \frac{A_{1}}{\delta_{1}{ }^{2}} \psi\left(\delta_{1}\right)\left(e^{-\delta_{1}{ }^{2} F o}-1\right)
$$

As at the stone-pulp interface (Eq. (21)):

$$
Y \approx A_{1} e^{-\delta_{1}^{2} F o}
$$

And the average power needed to cool the fruit up to the dimensionless temperature difference $Y$ can be approximated as:

$$
\overline{\dot{Q}}(t) \approx \dot{Q_{0}} \psi\left(\delta_{1}\right) \frac{A_{1}-Y}{\ln A_{1}-\ln Y}
$$

\subsubsection{Asymptotic dimensionless slope $\delta_{1}{ }^{2}$}


In practice, the first eigenvalue $\left(\delta_{1}\right)$ of the transcendental Eq. (9) is one of the most important parameter in the process, as knowledge of it allows us to know the surface heat transfer coefficient. Therefore, $-\delta_{1}{ }^{2}$ is the absolute slope of the linear portion of the cooling/heating curve in semi-logarithmic scale:

$$
\delta_{1}^{2}=-\frac{d(\ln Y)}{d(F o)}
$$

\subsubsection{Asymptote $B i \rightarrow 0$}

As seen in Fig. 2, the plot of $\delta_{1}$ vs. $B i$ is similar to that of a solid sphere (also drawn) but higher in value. When $B i \rightarrow 0, \delta_{1} \rightarrow 0$ as well, and its values can be estimated by considering the expressions derived from Cuesta et al. (2012) (see Appendix C):

$$
\delta_{1}^{2} \approx \frac{M_{1}{ }^{2}}{\left(1-x_{a}\right)} \cdot \frac{B i}{B i\left(1-x_{a}\right)\left(M_{1}+2 x_{a}-2\right)+x_{a} M_{1}{ }^{2}+2\left(1-x_{a}\right)^{2}}
$$

With:

$$
M_{1}=(\pi / 2)^{2}
$$

The maximum deviation of this equation is around $4 \%$ for $B i \leq 5$ and $0 \leq x_{a} \leq$ 0.9. The standard deviation is $1.44 \%$. If $B i \leq 1$ the maximum deviation is $\leq 2.5 \%$, the standard deviation being $<0.7 \%$.

Eq. (27) is also valid for $x_{a}=0$ (solid sphere):

$$
\delta_{1}^{2} \approx(\pi / 2)^{4} \frac{B i}{B i\left[(\pi / 2)^{2}-2\right]+2}
$$

Approximation (28) greatly improves the approximation seen in Cuesta and Lamúa (1995), which for the sphere can be written:

$$
\delta_{1}{ }^{2} \approx 6 \frac{B i}{B i+2}
$$

Indeed, (28) leads to a deviation $<3 \%$ for $B i \leq 5$ and to a deviation $<1 \%$ for $B i<3$. In the case of Eq. (29) they only had these deviations for $B i<0.25$. 


\subsubsection{Estimation of maximum values of $\delta_{n}$}

The maximum slope $\delta_{1, \text { Max }}^{2}$ depends only on the shape of the object. In our case, since the geometry is spherical, it depends only on the size of the stone. Furthermore, the values $\delta_{1, \text { Max }}^{2}$ can be approximated as in the above case for each particular value of $x_{a}$ by considering the expressions derived from the work of Cuesta et al. (2012). The approximate expression (see Appendix C) is as follows:

$$
\delta_{n, M a x}^{2} \approx \frac{1}{\left(1-x_{a}\right)^{2}} \times \frac{M_{n}^{2}}{M_{n}-2\left(1-x_{a}\right)}
$$

Or:

$$
\begin{aligned}
& \delta_{n, \text { Max }} \approx \frac{1}{\left(1-x_{a}\right)} \times \frac{M_{n}}{\sqrt{M_{n}-2\left(1-x_{a}\right)}} \\
& \text { For } n=1, M_{1}=(\pi / 2)^{2} \text { and: } \\
& \delta_{1, \text { Max }}^{2} \approx \frac{1}{\left(1-x_{a}\right)^{2}} \times \frac{(\pi / 2)^{4}}{(\pi / 2)^{2}-2\left(1-x_{a}\right)}
\end{aligned}
$$

Or:

$$
\delta_{1, \operatorname{Max}} \approx \frac{1}{\left(1-x_{a}\right)} \times \frac{(\pi / 2)^{2}}{\sqrt{(\pi / 2)^{2}-2\left(1-x_{a}\right)}}
$$

Eq. (27) includes Eq. (32) for $B i \rightarrow \infty$.

Fig. 3 shows $\delta_{1, \operatorname{Max}}$ obtained with approximation (33), the exact value (Eq. (11)), and the deviation (\%): 


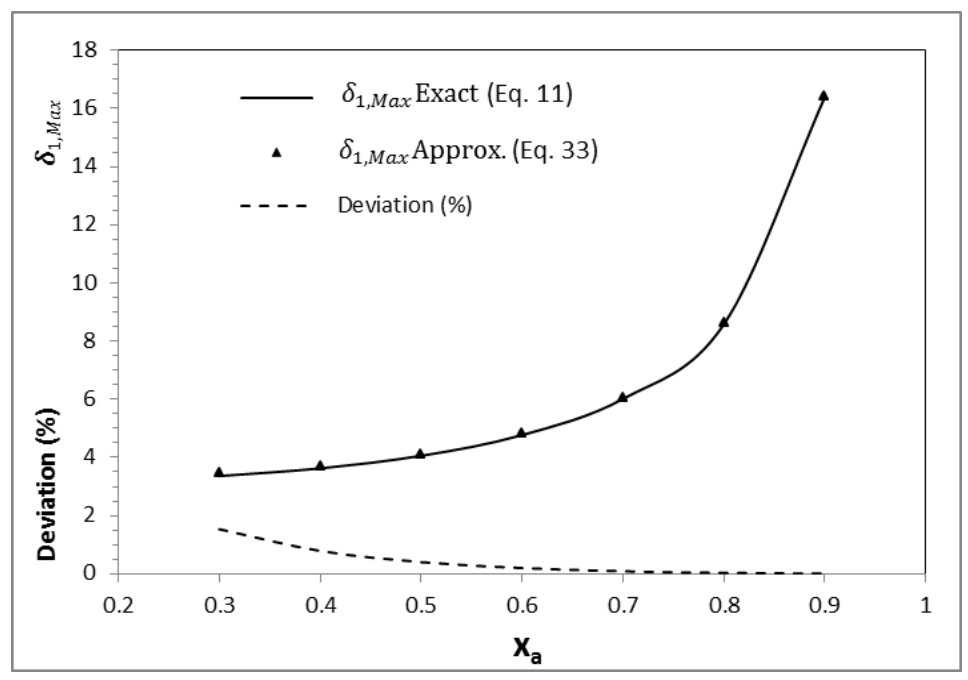

Fig. 3. Comparison of $\delta_{1, \operatorname{Max}}$ obtained with approximation (33) and the exact value (Equation 11). The deviation (\%) is also shown.

\subsubsection{Cooling/heating times}

As in the solid sphere, the time required to reach a dimensionless temperature $Y_{a}$ at the stone-pulp interface is:

$$
F o_{Y_{a}} \approx \frac{\ln A_{1}-\ln Y_{a}}{\delta_{1}{ }^{2}}=\frac{\ln \left[\frac{A_{1}}{Y_{a}}\right]}{\delta_{1}{ }^{2}}
$$

In the case of mass average temperature, $A_{1}$ should be substituted by $\bar{A}_{n}=$ $A_{n} \bar{\psi}\left(\delta_{n}\right)($ Eq. (14)).

If $B i \rightarrow \infty$, the slope attains its maximum $\left(-\delta_{1, \text { Max }}^{2}\right)$. Therefore the dimensionless time required to reach $Y_{a}$ is minimized:

$$
F o_{Y_{a}, \min } \approx \frac{\ln \left(A_{1, \infty} / Y_{a}\right)}{\delta_{1, \operatorname{Max}}^{2}}
$$

As in the case of $\delta_{1, \operatorname{Max}}$, when $B i \rightarrow \infty$ the dimensionless cooling/heating time $\left(F o_{Y_{a}, \min }\right)$ is indicated by the subscript " $m i n$ " to emphasize that it is the absolute 
minimum dimensionless time necessary to achieve the dimensionless temperature difference $Y_{a}$, as the surface heat transfer coefficient is considered to be infinite.

\subsubsection{Indirect measurement of thermal diffusivity and surface heat transfer coefficient}

According to Cuesta et al. (1990), Erdoğdu (2005, 2008), Erdoğdu et al. (2014), and Uyar and Erdoğdu (2012), the experimental lag factor $A_{1}$ allows us to determine the thermal diffusivity and the Biot number. In fact, the time-temperature history of the product at the thermal center (stone-pulp contact) can be transformed into a table $\left\{Y_{i}, t_{i}(s)\right\}$, where $Y_{i}=\left(T_{i}-T_{e x}\right) /\left(T_{0}-T_{e x}\right)$, so the linear regression to the linear portion of its graph in semi-logarithmic coordinates can be obtained:

$$
\ln Y \approx \ln A_{1, \exp }-S \cdot t
$$

Where, by identifying with (22), the lag factor $A_{1}$ is directly determined ( $A_{1}=$ $A_{1, \exp }$ ), and, by identifying with (23) and resolving, $a$ is deduced as well:

$$
a=\frac{S R^{2}}{\delta_{1}^{2}}
$$

The experimental $\delta_{1}$ can be determined by a trial and error method, and therefore the Biot number (that is, the dimensionless surface heat transfer coefficient) and the thermal diffusivity can also be determined. In fact, from a trial value $\delta_{1}$, the Biot number that corresponds to this trial $\delta_{1}$ can be deduced by resolving $B i$ in Eq. (9):

$$
B i=1+\frac{\delta_{1}^{2} x_{a}-m}{1+m x_{a}}
$$

Where:

$$
m=\frac{\delta_{1}}{\tan \left[\delta_{1}\left(1-x_{a}\right)\right]}
$$

And taking into account (8) and (10) for $n=1$ and $x=1$ :

$$
\psi\left(\delta_{1}\right)=\frac{\sin \left[\delta_{1}\left(1-x_{a}\right)\right]+\delta_{1} x_{a} \cos \left[\delta_{1}\left(1-x_{a}\right)\right]}{\delta_{1}}
$$




$$
A_{1}=\frac{2 B i \psi\left(\delta_{1}\right)}{\left[\psi\left(\delta_{1}\right)\right]^{2}\left[\delta_{1}^{2}+B i^{2}-B i\right]-\delta_{1}^{2} x_{a}^{3}}
$$

If $A_{1}=A_{1, \exp }$ (within the deviation considered), then $\delta_{1}, B i$ (i.e., the surface heat transfer coefficient) and the thermal diffusivity $a$ (by substituting in Eq. (37)) have been determined simultaneously, and therefore can be regarded as experimental.

If $A_{1} \neq A_{1, \exp }$ (within the deviation considered) then, by increasing or decreasing $\delta_{1}, A_{1}$ can be recalculated until the desired accuracy is obtained.

In practice, using a simple algorithm to accelerate the convergence, no more than 4 or 5 cycles of calculation are needed to determine $\delta_{1}, B i$, and $a$.

\section{Applications}

The purpose of this section is to illustrate the application of equations deduced in the preceding section, in the two different kinds of applications:

First: determination of thermal diffusivity and surface heat transfer coefficient. This requires measuring the temperature kinetics (Table $\left\{t(s)-T\left({ }^{\circ} \mathrm{C}\right)\right\}$ ).

Second: Estimation of design parameters (cooling/heating time, processing equipment). In this case we have to know the thermophysical parameters of the fruit and the surface heat transfer coefficient.

\subsection{Materials and methods}

The data used in this section are part of an actual experiment, which in turn is part of a broader work on prevention of browning of table olives by pre-cooling in cold water. The complete experiment was performed with twenty fruits. The fruits were cooled in order to evaluate the residual effect of the water pre-cooling on the rate of browning in a 
storage chamber at $4{ }^{\circ} \mathrm{C}$. Previously, the fruits were stabilized at $17^{\circ} \mathrm{C}$, and then dipped in a 4-liter bath with a mixture of water and crushed ice, stirred by a stirrer. At the time of introducing the olive samples, this mixture was stabilized at $0.4{ }^{\circ} \mathrm{C}$, approximately.

One of the twenty fruits above had a thermocouple in contact with the stone-pulp interface. The temperature was measured with a Testo 177-T4 AG data logger and TestLink SE309 software, with a resolution of $0.1{ }^{\circ} \mathrm{C}$ (from $-200{ }^{\circ} \mathrm{C}$ to $+200{ }^{\circ} \mathrm{C}$ ) and a reading precision of $1 \%$ up to $+200{ }^{\circ} \mathrm{C}$. We used two K-type bare wire thermocouple probes: one was inserted in the olive and the other measured the temperature of the water.

\subsection{Geometry and weight}

The olive sample weighed $10.44 \mathrm{~g}$, with an equatorial diameter $D=23.25 \mathrm{~mm}$ and a longitudinal diameter $L=32.44 \mathrm{~mm}$. After the complete experiment, the stone was measured. Its dimensions were as follows: weight $1.95 \mathrm{~g}$, equatorial diameter $d=$ $10.02 \mathrm{~mm}$, and longitudinal diameter $l=21.95 \mathrm{~mm}$.

\subsection{Equivalent sphere}

In order to reduce the shape of the sample to the model considered in this paper, the olive was considered as a sphere having the same volume as the actual olive. As it is very approximately an elongated ellipsoid, the radius of the equivalent sphere is:

$$
R=\frac{1}{2} \sqrt[3]{D^{2} L}=0.013 \mathrm{~m}
$$

And, similarly, the radius of the sphere equivalent to the stone is: 


$$
a=\frac{1}{2} \sqrt[3]{d^{2} l}=0.0065 \mathrm{~m}
$$

Hence, the ratio of radii is:

$$
x_{a}=0.501
$$

And the density of the pulp of the olive:

$$
\rho=1057.6 \mathrm{~kg} / \mathrm{m}^{3}
$$

\subsection{Application I. Determination of Biot number and thermal diffusivity}

Fig. 4a shows the semi-logarithmic-scale plot of the experimental values $\{\mathrm{Y}, \mathrm{t}(\mathrm{s})\}$, its linear portion, and the regression line of the linear zone. The lag factor $A_{1}$ and the cooling kinetic corresponding to $B i=\infty$ as the absolute limit case are also shown.

The linear regression is:

$$
\ln Y=0.2921-6.32310^{-3} t
$$

With

$$
R^{2}=0.999
$$

Therefore the experimental lag factor is:

$$
A_{1, \exp }=e^{0.2921}=1.339
$$

And the absolute slope:

$$
S=\frac{\delta_{1}^{2} a}{R^{2}}=6.32310^{-3} \mathrm{~s}^{-1}
$$

By applying the iterative process described above (Eqs. (37) to (41)), parameters $\delta_{1}$ and $B i$ are simultaneously obtained, and, by substituting $S$ in Eq. (37), the thermal diffusivity is determined:

$$
\begin{aligned}
& \delta_{1}=2.93 \\
& B i=4.46
\end{aligned}
$$




$$
a=1.23910^{-7} \mathrm{~m}^{2} \mathrm{~s}^{-1}
$$

As, at this point, the Biot number is already known $(B i=4.46)$, the roots of the transcendental boundary equation (Eq. 9) and the constants of the serial expansion (Eq. 10) can be calculated. Therefore, the complete theoretical time-temperature curve of this experiment can be drawn and compared with the experimental values. The result is shown in Fig. 4b (unbroken line), together with the experimental values (empty circles). The standard error between the experimental and theoretical $Y$ curves is \pm 0.01 (1.16\%).
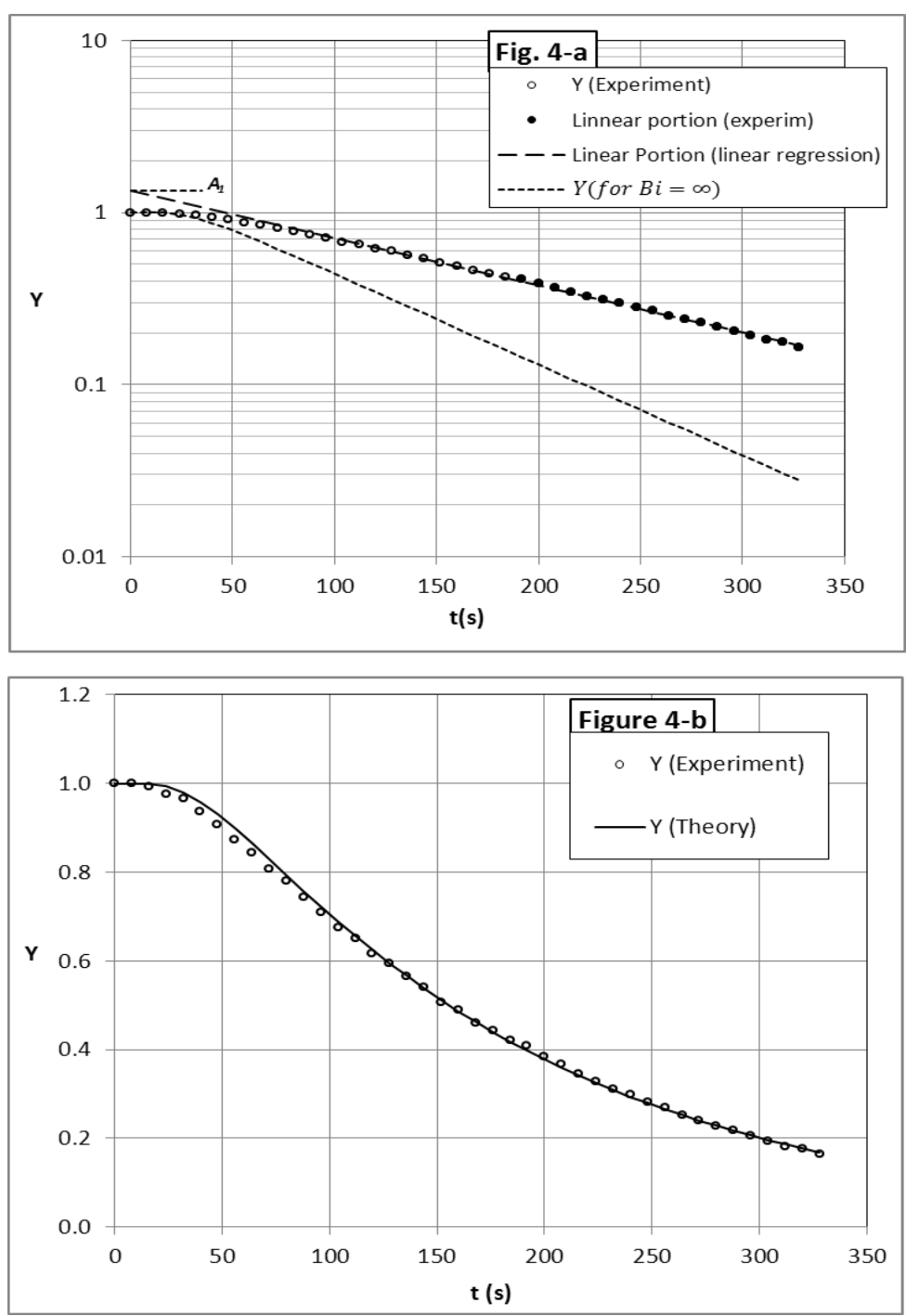

Fig. 4. (a): Semi-logarithmic-scale plot of the experimental values $\{Y, t(s)\}$ (hollow circles), linear portion (black circles) and the regression line of the linear zone (unbroken line), lag factor $A_{1}$ and cooling kinetic corresponding to $B i=\infty$ as the 


\section{absolute limit case (dotted line). (b): Complete theoretical series of the cooling process (unbroken line) compared with the experimental values (empty circles).}

On the other hand, from the chemical composition of the olive, the thermal diffusivity can be theoretically estimated according to ASHRAE (1998), Carson (2006), Carson et al. (2005, 2006), Choi and Okos (1986), Marcotte et al. (2008), Maroulis et al. (2002), and Murakami and Okos (1988), and compared with the diffusivity that has just been determined.

The chemical composition of the olive to be used for this work is as follows (averaged from Guillén et al. (1992), ASHRAE (1998), Ongen et al. (2005), and Wang et al. (2006)): $x_{\text {Water }}=75.97 \% ; x_{\text {Prot }}=4.37 \% ; x_{\text {fat }}=10.23 \% ; x_{\text {Ash }}=0.91 \% ; x_{\text {Carboh }}$ $=8.52 \%$ (by difference).

Thus the following values are calculated, which can be compared with those just obtained: thermal diffusivity $a=1.26810^{-7} \mathrm{~m}^{2} / \mathrm{s} \quad(2.4 \%)$ density $\rho=1038.2 \mathrm{~kg} / \mathrm{m}^{3}$ (1.8\%). By accepting the theoretical specific heat per unit mass $c_{p}=3608.6 \mathrm{~J} / \mathrm{kg} \mathrm{K}$, the conductivity can be obtained from the determined diffusivity:

$$
k=\rho c_{p} a=1057.6 \times 3608.6 \times 1.23910^{-7}=0.4730 \mathrm{~W} / \mathrm{m} \mathrm{K}
$$

This can be compared with that obtained from the chemical composition: $k=$ $0.4752 \mathrm{~W} / \mathrm{m} \mathrm{K}$ (deviation $0.5 \%$ ). Thus the estimated surface heat transfer coefficient is:

$$
h=\frac{k}{R} B i=162.3 \mathrm{~W} / \mathrm{m}^{2} \mathrm{~K}
$$

3.5. Application II. Prediction of cooling times and estimation of the heat to be extracted from the fruit 
An important issue in postharvest handling is the time that the fruit must stay in the hydro-cooler to achieve a certain temperature at the stone-pulp interface before moving to the next phase of its conservation or treatment. Thus, in this example the cooling time is estimated by Eq. (34), but approximating the slope by Eq. (27). It is assumed that the surface heat transfer coefficient $(h)$, the initial temperature of the fruit $\left(T_{0}\right)$, the water temperature in the hydro-cooler $\left(T_{e x}\right)$, and the nutritional composition of the fruit are known.

Since this is merely an illustrative example, we take the same case in reverse, and we could enunciate it as follows: In a water tank in which $T_{e x}=0.46^{\circ} \mathrm{C}$, the olive described in the Geometry and weight subsection of the above example has to be cooled from the initial temperature $T_{0}=17.7^{\circ} \mathrm{C}$ to the final temperature $T=4{ }^{\circ} \mathrm{C}$ at the stonepulp interface ( $Y=0.205$ ). The surface heat transfer coefficient is $h \approx 162 \mathrm{~W} / \mathrm{m}^{2} \mathrm{~K}$. How long does it take to reach that temperature? What is the average temperature at that moment?

As seen in the Equivalent sphere subsection, the radius of the equivalent sphere is $R=0.013 \mathrm{~m}$, the ratio of the stone radius to the pulp radius is $x_{a}=0.501$, and, as seen in Application I, from the nutritional composition the theoretical thermophysical parameters are: thermal diffusivity $a=1.26810^{-7} \mathrm{~m}^{2} / \mathrm{s}$, density $\rho=1038.2 \mathrm{~kg} / \mathrm{m}^{3}$, specific heat per unit mass $c_{p}=3608.6 \mathrm{~J} / \mathrm{kg} \mathrm{K}$, and conductivity $k=0.4752 \mathrm{~W} / \mathrm{m} \mathrm{K}$. As the surface heat transfer coefficient is $h=162 \mathrm{~W} / \mathrm{m}^{2} \mathrm{~K}$, the Biot number is:

$$
B i=\frac{162 \cdot 0.013}{0.4752}=4.43
$$

So, by approximating the slope with Eq. (27), and by introducing this in Eqs. (40) and (41):

$$
\delta_{1}^{2} \approx 7.95 \rightarrow \psi\left(\delta_{1}\right)=0.432 \text { and } A_{1}=1.155
$$

And by substitution in Equation (34): 


$$
F o_{Y_{a}} \approx 0.217
$$

So the estimated time (in seconds) is:

$$
t_{Y_{a}} \approx \frac{R^{2}}{a} F o_{Y}=296 \mathrm{~s}
$$

The time deduced from the linear regression is $t=297 \mathrm{~s}$. So the deviation is $0.23 \%$

The mass average temperature of the pulp at that moment is (by replacing $A_{1}$ in Eq.

(34) by $\bar{A}_{1}=A_{1} \bar{\psi}$, rearranging terms, and clearing $\bar{Y}_{a}$ :

$$
\bar{Y}_{a} \approx \bar{A}_{1} \exp \left(-\delta_{1}^{2} F o_{Y_{a}}\right) \approx 0.1
$$

That is:

$$
\bar{T}=T_{e x}+\bar{Y}_{a}\left(T_{0}-T_{e x}\right) \approx 2^{\circ} \mathrm{C}
$$

The estimated power required to cool the fruit would be (Eqs. (16) and (26)):

$$
\begin{aligned}
& \dot{Q}_{0}=4 \pi \cdot(0.013)^{2} \cdot 162 \cdot 17.24=5.922 \mathrm{~W} \\
& \dot{Q}(t) \approx 1.40 \mathrm{~W}(6 \% \text { deviation from complete series })
\end{aligned}
$$

Considering that this specimen of Gordal olive weighed $10.44 \mathrm{~g}$, the average calorific value would be $134.6 \mathrm{~W} / \mathrm{Kg}$, so that for a load of $1 \mathrm{Tm}$ (for example) the value would be: $\dot{Q}(t) \approx 135 \mathrm{~kW}$.

\section{Conclusion}

A Fourier series solution has been deduced for heat conduction in stone fruits for the kinetics of temperature in boundary conditions of the third kind with a constant external temperature. On the basis of the linear portion of the complete solution, an algorithm is proposed to directly determine the thermal diffusivity, the Biot number, and the first eigenvalue of the transcendental boundary equation. If the specific heat per unit mass is 
known, both the thermal conductivity and the surface heat transfer coefficient are also determined. On the basis of the new transcendental boundary equations and from the exact numerical series for the determination of the Biot number as seen in a previous paper, the first approximations are derived for the first eigenvalue of the transcendental boundary equation (that is, the slope of the linear portion in semi-logarithmic plot), and thus for the estimation of cooling times and the heat extracted from the fruit.

\section{Acknowledgments}

The authors wish to thank the project entitled "Integration of cooling and antioxidants technologies to control the 'molestado' in table olives collected by mechanized means” supported by INTERACEITUNA and the Spanish Ministry of Environment, Rural and Marine (MARM). The authors dedicate this work to the memory of Olga Ferrera. 


\section{Nomenclature}

$A_{n}=$ Series expansion constants

$\bar{A}_{n}=$ Series expansion constants (mass average)

$a=$ Radius of the stone $(\mathrm{m})$

$B i=h \cdot R / k=$ Biot number

$c=$ Specific heat capacity $\left(\mathrm{Wkg}^{-1} \mathrm{~K}^{-1}\right)$

$F_{O}=\alpha \cdot t / R^{2}=$ Fourier number

$F o_{Y}=$ Fourier number required to reach a dimensionless temperature $Y$

$h=$ Surface heat transfer coefficient $\left(\mathrm{Wm}^{-2} \mathrm{~K}^{-1}\right)$

$k=$ Thermal conductivity $\left(\mathrm{Wm}^{-1} \mathrm{~K}^{-1}\right)$

$m$ = Intermediate variable appearing in Eq. (39)

$M_{n}=[(n-1 / 2) \pi]^{2}=$ Constants appearing in Appendix C, Eq. (C-4)

(dimensionless)

$Q(t)=$ Total energy extracted up to the moment $t$

$\dot{Q}=$ Heat flow transferred through the surface at the moment $t$

$R=$ Radius of the body $(\mathrm{m})$

$r=$ Radius from the center of the fruit (m)

$S=$ Surface $\left(\mathrm{m}^{2}\right)$

$S=$ In Eq. (22): Absolute slope of the linear portion in semi-logarithmic scale

$\left(\mathrm{s}^{-1}\right)$

$t=$ Time (s)

$T=$ Temperature $\left({ }^{\circ} \mathrm{C}\right)$

$T_{e x}=$ Temperature of the medium $\left({ }^{\circ} \mathrm{C}\right)$

$\Delta T_{0}=\left(T_{0}-T_{e x}\right)$

$V=$ Volume $\left(\mathrm{m}^{3}\right)$ 
$x=r / R=$ Dimensionless distance from the center

$x_{a}=a / R=$ Dimensionless distance of the stone-pulp contact surface

$Y=\left(T-T_{e x}\right) /\left(T_{0}-T_{e x}\right)=$ Dimensionless ratio of temperature difference

$\bar{Y}=\left(\bar{T}-T_{e x}\right) /\left(T_{0}-T_{e x}\right)=$ Dimensionless ratio of mass average temperature difference

\section{Greek letters}

$\alpha=k /(\rho \cdot c)=$ Thermal diffusivity $\left(\mathrm{m}^{2} \mathrm{~s}^{-1}\right)$

$\delta^{2}=$ (Minus) slope of the linear portion on semi-logarithmic scale (Dimensionless)

$\delta_{n}{ }^{2}=$ Solutions to the transcendental equation of boundary condition (Dimensionless)

$\delta_{n, \operatorname{Max}}=\delta_{n}$ for $\mathrm{Bi} \rightarrow \infty$ (Dimensionless)

$\rho=\operatorname{Density}\left(\mathrm{kg} \mathrm{m}^{-3}\right)$

$\psi=\psi\left(\delta_{n} x\right)=$ The spatial component of the solution in Fourier series expansion

\section{Subscripts}

$0=$ Value when $t=0$

$1=$ First term in infinite series

$a=$ Value on the stone-pulp contact surface

$\exp =$ Experimental

Max $=$ Maximum value that can be achieved

$\min =$ Minimum value that can be achieved

$n=$ Index in infinite series

$s f=$ Value at $r=R$

$\infty=$ For $B i \rightarrow \infty$ 


\section{References}

ASHRAE. (1998). Thermal properties of foods. ASHRAE Handbook - Refrigeration (SI), American Society of Heating, Refrigerating and Air Conditioning Engineers, Chapter 8, pp. 8.2.

Awuah, G. B., Ramaswamy, H. S., \& Simpson, B. K. (1995). Comparison of two methods for evaluating fluid-to-surface heat transfer coefficients. Food Research International, 28(3), 261-271. doi:10.1016/0963-9969(94)00041-6.

Becker, B. R., \& Fricke, B. A. (2002). Hydrocooling time estimation methods. Int. Commun. Heat and Mass Transfer, 29(2), 165-174. doi:10.1016/S07351933(02)00307-X.

Carslaw, H. S. \& Jaeger, J. C. (1959). Conduction of Heat in Solids (2nd. Ed). Pp. 246250). Clarendon Press, Oxford.

Carson, J. K. (2006). Review of effective thermal conductivity models for foods. International Journal of Refrigeration, 29, 958-967. doi:10.1016/j.ijrefrig.2006.03.016.

Carson, J. K., Lovatt, S. J., Tanner, D. J., \& Cleland, A. C. (2005). Thermal conductivity bounds for isotropic, porous materials. International Journal of Heat and Mass Transfer, 48, 2150-2158. doi:10.1016/j.ijheatmasstransfer.2004.12.032.

Carson, J. K., Lovatt, S. J., Tanner, D. J., \& Cleland, A. C. (2006). Predicting the effective thermal conductivity of unfrozen, porous foods. Journal of Food Engineering, 75, 297-307. doi:10.1016/j.jfoodeng.2005.04.021.

Choi Y., \& Okos, M. R. (1986). Effects of temperature and composition on the thermal properties of foods. In L. Maguer \& P. Jelen (Eds.), Food Engineering and Process Applications: Transport Phenomenon (Vol. 1, pp. 93-116). Elsevier, New York. 
Cinquanta, L., Di Matteo, M., \& Estia, M. (2002). Physical pre-treatment of plums (Prunus domestica). Part 2. Effect on the quality characteristics of different prune cultivars. Food Chemistry, 79(2) 233-238. doi:10.1016/S0308-8146(02)00138-3.

Cleland, A. C. \& Earle, R. L. (1982). A simple method for prediction of heating and cooling rates in solids of various shapes. International Journal of Refrigeration, 5, 98-106. doi:10.1016/0140-7007(82)90084-6.

Cuesta F. J., Lamúa M., \& Moreno J. (1990). Graphical calculation of half-cooling times. International Journal of Refrigeration, 13, 317-324. doi:10.1016/01407007(90)90063-3.

Cuesta F. J. \& Lamúa M. (1995). Asymptotic modelling of the transient regime in heat conduction in solids with general geometry. Journal of Food Engineering, 24, 295320. doi:10.1016/0260-8774(95)90048-G.

Cuesta F. J. \& Lamúa M. (2002). Calculations of the minimum mean cooling/heating time of general-geometry solids. Journal of Food Engineering, 53(3), 259-271. doi:10.1016/S0260-8774(01)00164-9.

Cuesta F. J., Lamúa M., \& Alique R. (2012). A new exact numerical series for the determination of the Biot number: Application for the inverse estimation of the surface heat transfer coefficient in food processing. International Journal of Heat and Mass Transfer, 55, 4053-4062. doi:10.1016/j.ijheatmasstransfer.2012.03.047.

da Silva, W. P., Precker, J. W., e Silva, D. D. P. S., e Silva, C. D. P. S., \& Barbosa de Lima, A. G. (2009). Numerical simulation of diffusive processes in solids of revolution via the finite volume method and generalized coordinates. International Journal of Heat and Mass Transfer, 52, 4976-4985. doi: 10.1016/j.ijheatmasstransfer.2009.05.008. 
da Silva, W. P., e Silva, C. M. D. P. S., e Silva, D. D. P. S., de Araújo Neves, G., \& Barbosa de Lima, A. G. (2010). Mass and heat transfer study in solids of revolution via numerical simulations using finite volume method and generalized coordinates for the Cauchy boundary condition. Journal of Heat and Mass Transfer, 53, 1183-1194. doi: 10.1016/j.ijheatmasstransfer.2009.10.028.

Di Matteo, M., Cinquanta, L., Galiero, G., \& Crescitelli, S. (2000). Effect of a novel physical pretreatment process on the drying kinetics of seedless grapes. Journal of Food Engineering, 46, 83-89. doi:10.1016/S0260-8774(00)00071-6.

Di Matteo, M., Cinquanta, L., Galiero, G., \& Crescitelli, S. (2002). Physical pretreatment of plums (Prunus domestica). Part 1. Modelling the kinetics of drying. Food Chemistry, 79, 227-232. doi: 10.1016/S0308-8146(02)00137-1.

Di Matteo, M., Cinquanta, L., Galiero, G., \& Crescitelli, S. (2003). A mathematical model of mass transfer in spherical geometry: plum (Prunus domestica) drying. Journal of Food Engineering, 58, 183-192. doi: 10.1016/S0260-8774(02)00368-0.

Erdoğdu, F. (2005). Mathematical approaches for use of analytical solutions in experimental determination of heat and mass transfer parameters. Journal of Food Engineering, 68, 233-238. doi:10.1016/j.jfoodeng.2004.05.038.

Erdoğdu, F. (2008). A review on simultaneous determination of thermal diffusivity and heat transfer coefficient. Journal of Food Engineering, 86, 453-459. doi: 10.1016/j.jfoodeng.2007.10.019.

Erdoğdu, F., Linke, M., Praeger, U., Geyer, M., \& Schlüter O. (2014). Experimental determination of thermal conductivity and thermal diffusivity of whole green (unripe) and yellow (ripe) Cavendish bananas under cooling conditions. Journal of Food Engineering, 128, 46-52. doi:10.1016/j.jfoodeng.2013.12.010. 
Fabbri, A., Cevoli, C., Alessandrini, L., \& Romani, S. (2011). Numerical modeling of heat and mass transfer during coffee roasting process. Journal of Food Engineering, 105, 264-269. doi: 10.1016/j.jfoodeng.2011.02.030.

Gac, A. (1963). Détermination du refroidissement des denrées par réfrigération. In Proceedings of the XIth. International Congress of Refrigeration, II, 943-952. Karlsruhe, Germany.

Guillén, R., Heredia, A., Felizón, B., Jiménez, A., Montaño, A., \& Fernández-Bolaños, J. (1992). Fibre fraction carbohydrates in Olea europaea (Gordal and Manzanilla var.). Food Chemistry, 44, 173-178. doi:10.1016/0308-8146(92)90183-3.

Helal, M. M. (2012). Generalization of the integral transform method to nonlinear heatconduction problems in multilayered spherical media. Journal of King Saud University - Science, 24, 367-377. doi:10.1016/j.jksus.2012.01.002.

Hernández, Fca, Pinochet, J., Moreno, M. A., Martínez, J. J., \& Legua, P. (2010). Performance of Prunus rootstocks for apricot in Mediterranean conditions. Scientia Horticulturae, 124, 354-359. doi:10.1016/j.scienta.2010.01.020.

Hernández-Díaz, W. N., Ruiz-López, I. I., Salgado-Cervantes, M. A., RodríguezJimenes, G. C., \& García-Alvarado, M. A. (2008). Modeling heat and mass transfer during drying of green coffee beans using prolate spheroidal geometry. Journal of Food Engineering, 86, 1-9. doi:10.1016/j.jfoodeng.2007.08.025.

Jiménez-Jiménez, F., Castro-García, S., Blanco-Roldán, G. L., Ferguson, L., Rosa, U. A., \& Gil-Ribes, J. A. (2013). Table olive cultivar susceptibility to impact bruising. Postharvest Biology and Technology, 86, 100-106. doi: 10.1016/j.postharvbio.2013.06.024. 
Kondjoyan, A. (2006). A review on surface heat and mass transfer coefficients during air chilling and storage of food products. International Journal of Refrigeration, 29, 863-875. doi:10.1016/j.ijrefrig.2006.02.005.

Lin, Z., Cleland, A. C., Cleland, D. J., \& Serrallach, G. F. (1996a). A simple method for prediction of chilling times for objects of two dimensional irregular shape. International Journal of Refrigeration, 19, 95-106. doi:10.1016/01407007(95)00081-X.

Lin, Z., Cleland, A. C., Cleland, D. J., \& Serrallach, G.F. (1996b). A simple method for prediction of chilling times: extension to three-dimensional irregular shapes. International Journal of Refrigeration, 19, 107-114. doi:10.1016/01407007(95)00082-8.

Lin, Z., Cleland, A. C., Cleland, D. J., \& Serrallach, G. F. (2000). Erratum to “A simple method for prediction of chilling times: extension to three-dimensional irregular shapes” [International Journal of Refrigeration, 19 (1996) 107-114]. International Journal of Refrigeration, 23, 168. doi: 10.1016/S0140-7007(99)00060-2.

Marcotte, M., Taherian, A. R., \& Karimi, Y. (2008). Thermophysical properties of processed meat and poultry products. Journal of Food Engineering, 88, 315-322. doi:10.1016/j.jfoodeng.2008.02.016.

Maroulis, Z. B., Krokida, M. K., \& Rahman, M. S. (2002). A structural generic model to predict the effective thermal conductivity of fruits and vegetables during drying. Journal of Food Engineering, 52, 47-52. doi:10.1016/S0260-8774(01)00084-X.

Murakami, E. G., \& Okos, M. R. (1988). Measurement and prediction of thermal properties of foods. In R. P. Singh \& A. G. Medina (Eds.), Food properties and computer-aided engineering of food processing systems (Part 1, pp. 3-48). NATO ASI series - Kluwer Academic Publishers. Boston. 
Nilnont, W., Thepa, S., Janjai, S., Kasayapanand, N., Thamrongmas, C., \& Bala, B. K. (2012). Finite element simulation for coffee (Coffea arabica) drying. Food and Bioproducts Processing, 90, 341-350. doi:10.1016/j.fbp.2011.06.007.

Ongen, G., Sargın, S., Tetik, D. \& Köse, T. (2005). Hot air drying of green table olives. Food Technology and Biotechnology, 43, 181-187.

Pflug, I. J., Blaisdell, J. L., \& Kopelman, J. (1965). Developing temperature-time for objects that can be approximated by sphere, infinite plate or infinite cylinder. ASHRAE Transactions, 71, 238-248.

Ruiz-López, I. I., Cordova, A. V., Rodríguez-Jimenes, G. C., \& García-Alvarado, M. A. (2004). Moisture and temperature evolution during food drying: effect of variable properties. Journal of Food Engineering, 63, 117-124. doi:10.1016/S02608774(03)00290-5.

Ruiz-López, I. I., \& García-Alvarado, M. A. (2007). Analytical solution for food-drying kinetics considering shrinkage and variable diffusivity. Journal of Food Engineering, 79, 208-216. doi:10.1016/j.jfoodeng.2006.01.051.

Ruiz-López, I. I., Ruiz-Espinosa, H., Luna-Guevara, M. L., \& García-Alvarado, M. A. (2011). Modeling and simulation of heat and mass transfer during drying of solids with hemispherical shell geometry. Computers \& Chemical Engineering, 35, 191199. doi:10.1016/j.compchemeng.2010.05.007.

Ruiz-López, I. I., Ruiz-Espinosa, H., Arellanes-Lozada, P., Bárcenas-Pozos, M. E., \& García-Alvarado, M. A. (2012). Analytical model for variable moisture diffusivity estimation and drying simulation of shrinkable food products. Journal of Food Engineering, 108, 427-435. doi:10.1016/j.jfoodeng.2011.08.025. 
Sabarez, H. T. (2012). Computational modelling of the transport phenomena occurring during convective drying of prunes. Journal of Food Engineering, 111, 279-288. doi:10.1016/j.jfoodeng.2012.02.021.

Smith, R. E., Nelson, G. L., \& Henrickson, R. L. (1967). Analyses on transient heat transfer from anomalous shapes. Transactions of the ASAE, 10(2), 236-244.

Uyar, R., \& Erdoğdu, F. (2012). Numerical evaluation of spherical geometry approximation for heating and cooling of irregular shaped food products. Journal of Food Science, 77, E166-E175. doi:10.1111/j.1750-3841.2012.02769.x.

Van Beek, G., \& Meffert, H. F. Th. (1997). Letter to the editor. International Journal of Refrigeration, 20, 71. doi:10.1016/S0140-7007(97)00002-9.

Wang, J. F., Carson, J. K., North, M. F., \& Cleland, D. J. (2006). A new approach to the modeling of the effective thermal conductivity of heterogeneous materials. International Journal of Heat and Mass Transfer, 49, 3075-3083. doi: 10.1016/j.ijheatmasstransfer.2006.02.007.

Yilmaz, T. (1995). Equations for heating and cooling of bodies of various shapes. International Journal of Refrigeration, 18, 395-402. doi:10.1016/01407007(95)98162-E. 


\section{Supplementary material: Appendices A-C}

Supplementary material (Appendices A-C) related to this article can be found at...

\section{Appendix A. General solution}

As noted in the Temperature kinetics subsection, the non-dimensional heat conduction to be solved is:

$$
\frac{1}{x^{2}} \frac{\partial}{\partial x}\left(x^{2} \frac{\partial Y}{\partial x}\right)=\frac{\partial Y}{\partial F O}
$$

With the initial conditions:

$$
F O=0: Y_{0}=1
$$

And the following boundary conditions:

A) External surface: Boundary conditions of the third kind:

$$
\text { Fo }>0 ;\left[\frac{\partial Y}{\partial x}\right]_{x=1}=-B i Y_{S f}
$$

$B i$ being the Biot number.

B) The pulp-to-stone contact surface:

$$
\text { Fo }>0 ; x=x_{a}:\left[\frac{\partial Y}{\partial x}\right]_{x=x_{a}}=0
$$

As we can see, for example in Carslaw and Jaeger (1959), in these conditions (A.1) admits a separate-variable solution in the form:

$$
Y(x, F O)=e^{-\delta^{2} F o} \psi(\delta x)
$$

Where $\psi(\delta x)$ satisfies the radial equation:

$$
\frac{1}{x^{2}} \frac{\partial}{\partial x}\left(x^{2} \frac{\partial \psi(\delta x)}{\partial x}\right)=-\delta^{2} \psi(\delta x)
$$

which must satisfy the condition (A.4): 
Fo $>0 ; x=x_{a}:\left[\frac{\partial \psi(\delta x)}{\partial x}\right]_{x=x_{a}}=0$

In general, the solution $\psi(\delta x)$ can be written in the form (Carslaw and Jaeger, 1959):

$\psi(\delta x)=M \frac{\sin (\delta x)}{x}+N \frac{\cos (\delta x)}{x}$

In the case of the homogeneous solid sphere, the symmetry condition in $x=0$ rules out the first addend of the second term, but in the present case the symmetry does not occur explicitly but in the context of a core having a radius $x_{a}=a / R$. As, by condition (A.4), the derivative vanishes at $x=x_{a}$, for convenience we can write the solution so that it includes the difference $\left(x-x_{a}\right)$. This is possible because all that changes is the value of the constants $M$ and $N$. Thus, the solution can be written in the form:

$$
\psi(\delta x)=M \frac{\sin \left[\delta\left(x-x_{a}\right)\right]}{x}+N \frac{\cos \left[\delta\left(x-x_{a}\right)\right]}{x}
$$

This satisfies the differential equation (A.5), as can be verified by simply substituting and regrouping its terms. The derivative of function (A.6) is:

$$
\frac{\partial \psi(\delta x)}{\partial x}=M \frac{\delta \cos \left[\delta\left(x-x_{a}\right)\right]}{x}-N \frac{\delta \sin \left[\delta\left(x-x_{a}\right)\right]}{x}-\frac{\psi(\delta x)}{x}
$$

In $x=x_{a}, \sin \left[\delta\left(x-x_{a}\right)\right]=0$ and $\cos \left[\delta\left(x-x_{a}\right)\right]=1$, and hence:

$$
\psi\left(\delta x_{a}\right)=N / x_{a}
$$

The aim is for the contact surface with the stone to play the same role as is played by the thermal center in the homogeneous solid sphere; therefore, we take $N$ so that:

$$
\psi\left(\delta x_{a}\right)=1
$$


which is possible because the constants of the complete solution are calculated afterwards. That is:

$$
N=x_{a}
$$

Substituting in (A. 6), taking into account condition (A.4), and resolving $M$ :

$$
M=1 / \delta
$$

Thus, the function in general is left as:

$$
\psi(\delta x)=\frac{\sin \left[\delta\left(x-x_{a}\right)\right]+\delta x_{a} \cos \left[\delta\left(x-x_{a}\right)\right]}{\delta x}
$$

As in the case of the homogeneous solid sphere, the number of possible solutions is infinite, and so the solution can be written in the form of a Fourier series:

$$
Y(x, F o)=\sum A_{n} \psi\left(\delta_{n} x\right) e^{-\delta_{n}^{2} F o}
$$

Where the coefficients $A_{n}$ depend on the initial and boundary conditions imposed, as we can see in Appendix B. 


\section{Appendix B. Roots of the boundary equation $\left(\delta_{n}\right)$ and series expansion}

constants $\left(A_{n}\right)$

As seen in Appendix A, the general solution of the heat conduction equation (2) can be expressed as a Fourier series:

$$
Y(x, F O)=Y=\sum A_{n} \psi\left(\delta_{n} x\right) e^{-\delta_{n}^{2} F o}
$$

Where $\psi\left(\delta_{n} x\right)$ is:

$$
\psi\left(\delta_{n} x\right)=\frac{\sin \left[\delta_{n}\left(x-x_{a}\right)\right]+\delta_{n} x_{a} \cos \left[\delta_{n}\left(x-x_{a}\right)\right]}{\delta_{n} x}
$$

With the boundary conditions of the third kind:

$$
\text { Fo }>0 ;\left[\frac{\partial Y}{\partial x}\right]_{x=1}=-B i Y_{S f}
$$

Substituting (B.2) into (B.3) and rearranging terms, we get the boundary equation from which the eigenvalues $\delta_{n}$ can be obtained:

$$
\delta_{n}=\tan \left[\delta_{n}\left(1-x_{a}\right)\right] \frac{1-B i+\delta_{n}^{2} x_{a}}{1+x_{a}(B i-1)}
$$

As in the case of heat transfer in a homogeneous isotropic solid body (Carslaw \& Jaeger, 1959), to calculate the constants $A_{n}$ we multiply both sides of equation (B.1) by $\left[x^{\Gamma} \psi(\mu x)\right]$ and integrate between $x=x_{a}$ and $x=1$. We get:

$$
A_{n}=\frac{2 B i \psi\left(\delta_{n}\right)}{\left[\psi\left(\delta_{n}\right)\right]^{2}\left(\delta_{n}^{2}+B i^{2}-B i\right)-\delta_{n}^{2} x_{a}^{3}}
$$

When $B i \rightarrow \infty$, and identifying by the subscript $\infty$ values of $A_{n}$ for this limit and by the subscript "Max" values of $\delta_{n}$ to indicate that it is the maximum value that can be achieved, (B.4) and (B.5) become, respectively:

$$
\tan \left[\delta_{n, \infty}\left(1-x_{a}\right)\right]+\delta_{n, \infty} x_{a}=0
$$

And 


$$
A_{n, \infty}=\frac{-2}{\cos \left[\delta_{n, \infty}\left(1-x_{a}\right)\right]} \frac{1}{\left(1+\delta_{n, \infty}^{2} x_{a}^{2}-\delta_{n, \infty}^{2} x_{a}^{3}\right)}
$$

for the mass average case

$$
\bar{A}_{n}=A_{n} \bar{\psi}\left(\delta_{n}\right)
$$

Where, if $V$ is the volume:

$\bar{\psi}\left(\delta_{n}\right)=\frac{1}{V} \int \psi\left(\delta_{n}\right) d V$

The expansion constants become:

$\bar{A}_{n}=\frac{6 B i^{2}}{\delta_{n}^{2}\left(1-x_{a}^{3}\right)} \frac{\left[\psi\left(\delta_{n}\right)\right]^{2}}{\left.\left[\delta_{n}\right)\right]^{2}\left[\delta_{n}^{2}+B i^{2}-B i\right]-\delta_{n}^{2} x_{a}^{3}}$

And when $B i \rightarrow \infty$ :

$$
\bar{A}_{n, \infty}=\frac{6}{\delta_{n, \infty}^{2}\left(1-x_{a}^{3}\right)\left(1+\delta_{n, \infty}^{2} x_{a}^{2}-\delta_{n, \infty}^{2} x_{a}^{3}\right)}
$$




\section{Appendix C. Asymptotic approximations}

\section{Asymptote $B \boldsymbol{i} \rightarrow \mathbf{0}$}

The values $\delta_{n}$ can be estimated by considering the expressions derived from Cuesta et al. (2012). In fact, the transcendental boundary equation is (Eq. 9):

$$
\delta_{n}=\frac{1-B i+\delta_{n}^{2} x_{a}}{1+x_{a}(B i-1)} \tan \left[\delta_{n}\left(1-x_{a}\right)\right]
$$

However, as seen in Cuesta et al. (2012), the function $\tan \alpha$ admits the following serial expansion:

$$
\tan \alpha=\alpha\left[1+\sum_{k=1}^{\infty} \frac{2}{[(k-1 / 2) \pi]^{2}} \times \frac{\alpha^{2}}{[(k-1 / 2) \pi]^{2}-\alpha^{2}}\right]
$$

This must also be valid for:

$$
\alpha=\delta_{n}\left(1-x_{a}\right)
$$

Thus, if we insert (C.2) and (C.3) in (C.1) and, for simplicity’s sake, we write:

$$
M_{k}=[(k-1 / 2) \pi]^{2}
$$

This leaves:

$$
\frac{\alpha}{\left(1-x_{a}\right)}=\frac{\left[1-B i+\delta_{n}^{2} x_{a}\right]}{\left[1+x_{a}(B i-1)\right]} \alpha\left[1+\sum_{k=1}^{\infty} \frac{2}{M_{k}} \times \frac{\alpha^{2}}{M_{k}-\alpha^{2}}\right]
$$

That is:

$$
\frac{1}{\left(1-x_{a}\right)}=\frac{\left[1-B i+\delta_{n}^{2} x_{a}\right]}{\left[1+x_{a}(B i-1)\right]}\left[1+2 \alpha^{2} \sum_{k=1}^{\infty} \frac{1}{M_{k}\left(M_{k}-\alpha^{2}\right)}\right]
$$

In this section we want to know the slope (in semi-logarithmic scale) of the cooling/heating curve; that is, the first eigenvalue $\left(\delta_{1}\right)$ of equation (C.1), when $B i \rightarrow 0$. In this case $\delta_{1} \rightarrow 0$ as well, so we can retain only the first term of the series. Therefore we can write:

$$
\frac{1}{\left(1-x_{a}\right)} \approx \frac{\left[1-B i+\delta_{1}^{2} x_{a}\right]}{\left[1+x_{a}(B i-1)\right]}\left[1+\frac{2}{M_{1}} \times \frac{\alpha^{2}}{M_{1}-\alpha^{2}}\right]
$$

Where: 


$$
M_{1}=(\pi / 2)^{2}
$$

Rearranging terms, taking into account (C.3), solving for $\delta_{1}^{2}$, and disregarding the terms in $\delta_{1}^{4}$ :

$$
\delta_{1}^{2} \approx \frac{M_{1}{ }^{2}}{\left(1-x_{a}\right)} \cdot \frac{B i}{B i\left(1-x_{a}\right)\left(M_{1}+2 x_{a}-2\right)+x_{a} M_{1}^{2}+2\left(1-x_{a}\right)^{2}}
$$

When $x_{a}=0$ (solid sphere):

$$
\delta_{1}^{2} \approx(\pi / 2)^{4} \frac{B i}{B i\left[(\pi / 2)^{2}-2\right]+2}
$$

In the case of the solid sphere, the approximation (C.6) greatly improves the approximation derived from the development in Taylor series (see, for example, Cuesta and Lamúa 1995), which, particularized for the sphere, could be written as follows:

$$
\delta_{1}^{2} \approx 6 \frac{B i}{B i+2}
$$

Indeed, (C.6) leads to a deviation $\leq 3 \%$ for $\mathrm{Bi} \leq 5$; and $\leq 1 \%$ for $\mathrm{Bi} \leq 2.75$. In the case of (C.7) they had only these deviations for $\mathrm{Bi} \leq 0.25$.

\section{Estimation of maximum values of $\delta_{n}$}

Similarly to the previous paragraph, when $B i \rightarrow \infty$ the values $\delta_{n, \text { Max }}$ can also be estimated by introducing Eq. (C.2) into equation (11) written as:

$$
-\delta_{n, \operatorname{Max}} x_{a}=\tan \left[\delta_{n, \operatorname{Max}}\left(1-x_{a}\right)\right]
$$

In this case:

$$
\alpha=\delta_{n, \operatorname{Max}}\left(1-x_{a}\right)
$$

Thus, if we insert (C.8) into (C.2):

$$
\tan \delta_{n, \operatorname{Max}}\left(1-x_{a}\right)=\delta_{n, \operatorname{Max}}\left(1-x_{a}\right)\left[1+\sum_{k=1}^{\infty} \frac{2}{M_{k}} \times \frac{\alpha^{2}}{M_{k}-\alpha^{2}}\right]
$$

And Eq. (C.8) is:

$$
-\delta_{n, \operatorname{Max}} x_{a}=\delta_{n, \operatorname{Max}}\left(1-x_{a}\right)\left[1+\sum_{k=1}^{\infty} \frac{2}{M_{k}} \times \frac{\alpha^{2}}{M_{k}-\alpha^{2}}\right]
$$

Simplifying and rearranging terms: 


$$
\frac{-1}{\left(1-x_{a}\right)}=\sum_{k=1}^{\infty} \frac{2}{M_{k}} \times \frac{\delta_{n, \operatorname{Max}}^{2}\left(1-x_{a}\right)^{2}}{M_{k}-\delta_{n, \operatorname{Max}}^{2}\left(1-x_{a}\right)^{2}}
$$

Given that the series (C.2) is rapidly convergent, and, moreover, whatever the value of $\alpha$, it will always be between two successive values of $(k-1 / 2) \pi$, only this term should be considered in a first approximation. Therefore Eq. (C.9) can be written approximately in the form:

$$
\frac{-1}{\left(1-x_{a}\right)} \approx \frac{2}{M_{n}} \times \frac{\delta_{n, \operatorname{Max}}^{2}\left(1-x_{a}\right)^{2}}{M_{n}-\delta_{n, \text { Max }}^{2}\left(1-x_{a}\right)^{2}}
$$

From which $\delta_{1, \text { Max }}^{2}$ can be readily resolved, leaving:

$$
\delta_{n, M a x}^{2} \approx \frac{1}{\left(1-x_{a}\right)^{2}} \times \frac{M_{n}{ }^{2}}{M_{n}-2\left(1-x_{a}\right)}
$$

Or:

$$
\begin{aligned}
& \delta_{n, \text { Max }} \approx \frac{1}{\left(1-x_{a}\right)} \times \frac{M_{n}}{\sqrt{M_{n}-2\left(1-x_{a}\right)}} \\
& \text { For } n=1, M_{1}=(\pi / 2)^{2} \text { and: } \\
& \delta_{1, \text { Max }} \approx \frac{1}{\left(1-x_{a}\right)} \times \frac{(\pi / 2)^{2}}{\sqrt{(\pi / 2)^{2}-2\left(1-x_{a}\right)}}
\end{aligned}
$$

